\title{
PYROELECTRICITY IN NON-CENTRAL CRYSTALS
}

\author{
L.P. Pereverzeva, Yu.M. Poplavko, Yu.V. Prokopenko
}

Kiev Polytechnic Institute, 37 Peremogi av., 252056 Kiev, Ukraine

AND A.G. ChepilKo

Institute of Physics, AS Ukraine, 46 Nauki av., 252028 Kiev, Ukraine

(Received Oclober 5, 1992; revised version April 14, 1993)

Unit cell electric arrangement of piezoelectric crystals may be described by various multipole/octupole/dipole electric moments which corresponds to a three/lwo/one-dimensional intrinsic polarity respectively. Such a latent polar structure is totally self-compensated in piezoelectrics if they are mechanically free, but it is non-compensated in pyroelectrics and ferroelectrics. Uniform but anisotropic partial clamping destroys a total self-compensation of non-central crystal intrinsic polarity. This decompensation allows to observe a polar response that is a "dipole projection" $\Delta P_{i}$ from, broken by clamping, spatial polar arrangement of piezoelectrics.

PACS numbers: 77.70. $+\mathrm{a}, 77.80 . \mathrm{Bh}$

The basic crystal unit cell of any piezoelectric forms as an electrically asymmetric system. Its elemental electric arrangement is created by electronic and ionic forces and may be described by octupole/quadrupole/dipole moments or their complex spatial combination. Because of cooperative effect such a polar explanation of piezoelectric unit cell motivates in a bulk crystal a sort of "inherent", "latent" or "intrinsic" polarization directed on so-called "polar axes". In order to describe piezoelectric polarity let us agree about the terminology "intrinsic polarization". Such a definition is desirable in order to distinguish between planar or spatial-compensated multipolar moments of piezoelectric and non-compensated dipole moments, that is a spontaneous polarization of 10 pyroelectric classes of crystals. Thus the intrinsic polar structure is non-compensated in 10 pyroelectric classes of crystals, but it is exactly compensated in other 10 piezoelectric classes.

The change of temperature alters the ionic and electronic forces in piezoelectric unit cell. The thermal disorder in crystal increases or decreases which results in a change of values of the octupole/quadrupole/dipole electric moments. But if the crystals are mechanically free, only in 10 piezoelectric classes of crystals one obtains pyroelectric response. An uniform change of temperature could not separate 
any charges in a piezoelectric crystal, because piezoelectrically transduced thermal strains are totally compensated. Nevertheless, we have found recently the conditions in which scalar (uniform) thermal or elastic influences induce pyroelectricity or volume piezoeffect in all 20 pyroelectric classes of crystals. It is the anisotropic limitation of thermal strain which we propose to prove the polar motivation in piezoelectrics which are not pyroelectrics. The partial clamping may be realized experimentally $[1,2]$.

The unit cell of gallium arsenide crystal contains 8 atoms and such a cell has the intrinsic polarity which is totally compensated because its four polar axes of threefold symmetry are crossing at the angle of $109^{\circ} 28^{\prime}$. If one cuts the crystal in a special way and provides anisotropic limitation of thermal strain, the artificial pyroelectric response could be obtained. The $100 \mu \mathrm{m}$ thick GaAs plate produces about $2 \mathrm{~V} / \mathrm{K}$, but it was established that some of $\mathrm{A}^{\mathrm{III}} \mathrm{B}^{\mathrm{V}}$ crystals have 10 times larger responsibility and they can form solid solutions with GaAs.

These data were obtained by a dynamic experiment with $\mathrm{CO}_{2}$ laser. The laser radiation was modulated in frequency range up to $1 \mathrm{MIIz}[2,3]$. Artificial pyroelectric response of piezoelectrics was calculated from thermodynamic equations $[4,5]$. For the crystals of $\overline{4} 3 m$ point symmetry the "pyroelectric" constant is

$$
\gamma_{111}=\frac{2 \sqrt{3} \alpha d}{4 S_{11}+8 S_{12}+S_{44}}
$$

where $d$ is piezoelectric constant, $\alpha$ is thermal expansion coefficient and $S_{k l}$ are compliances. Theoretical calculation shows $\gamma=1.5 \mu \mathrm{C} \mathrm{m}^{-2} \mathrm{~K}^{-1}$ for GaAs. In piezoelectric quartz from analogous formula $\gamma=2.6 \mu \mathrm{C} \mathrm{m}^{-2} \mathrm{~K}^{-1}$. Results of these calculations are close to the experimental ones and they coincide with a maximal response, but to describe "pyroelectricity" of slanting cuts one may use the indicatory surfaces shown in [4].

The "pyroelectric" response depends on temperature change of intrinsic polarization which decreases when the temperature rises. Spatial self-compensation of four polar axes (that is a three-dimensional case) corresponds to GaAs. Consequently, in special conditions uniform elastic or thermal perturbations may induce a volume piezoeffect or pyroelectric response correspondingly. The last effect may be named "thermopiezoelectric" response (TPER).

The maximum TPER coefficient was obtained in our experiments in a wellknown piezoelectric crystal $\mathrm{Bi}_{12} \mathrm{SiO}_{20}$ which belongs to a cubic 23 point group of symmetry. This crystal has a piezoelectric constant $d_{14}=4 \times 10^{-11} \mathrm{~m} / \mathrm{V}$ and a thermal expansion coefficient $\alpha=14 \times 10^{-6} \mathrm{~K}^{-1}$. Thin plates $0.1-0.3 \mathrm{~mm}$ of [111]-cut of $\mathrm{Bi}_{12} \mathrm{SiO}_{20}$ were covered by silver electrodes and then were soldered on a fused silica substrate in which $\alpha \approx 0$. The substrate was also covered by silver. Such a sandwich revealed itself as a "pyroelectric crystal" with "pyroelectric" (TPER) coefficient $\gamma=30 \mu \mathrm{C} \mathrm{m}^{-2} \mathrm{~K}^{-1}$ which coincides with values of usual pyroelectric crystals.

We have got the TPER "pyrocoefficient" $\gamma=\mathrm{d} P / \mathrm{d} T$ and voltage sensitivity $S_{V}=\gamma / C_{V} \varepsilon_{0} \varepsilon$, where $C_{V}$ heat capacity per volume and $\varepsilon$ is dielectric permittivity. These parameters for various crystals were compared in Table I with $\gamma$ and $S_{V}$ of standard pyroelectrics such as $\mathrm{LiNbO}_{3}$ and $\mathrm{LiTaO}_{3}$. In the last crystals [010]-cut shows TPER while [001]-cut characterizes usual pyroelectricity. 
TABLE I

Experimental data of TPER coefficients and voltage sensitivities for various piezoelectrics and two pyroelectrics.

\begin{tabular}{c|c|c|c}
\hline $\begin{array}{c}\text { Point } \\
\text { group }\end{array}$ & Crystal & $\begin{array}{c}\gamma_{i} \\
{\left[\mathrm{C} \mathrm{m}^{-2} \mathrm{~K}^{-1}\right]}\end{array}$ & $\begin{array}{c}S_{V} \\
{\left[\mathrm{~V} \mathrm{~m}^{2} \mathrm{~J}^{-1}\right]}\end{array}$ \\
\hline $23,[111]$ & $\mathrm{Bi}_{12} \mathrm{SiO}_{20}$ & 28 & 0.09 \\
$32,[100]$ & $\mathrm{SiO}_{2}$ & 2.6 & 0.035 \\
$\overline{4} 3 m,[111]$ & $\mathrm{GaAs}$ & 1.5 & 0.016 \\
$\overline{4} 2 m,[110]$ & $\mathrm{KDP}$ & 7.2 & 0.02 \\
$\overline{4} 2 m,[110]$ & $\mathrm{ADP}$ & 17 & 0.05 \\
$3 m,[010]$ & $\mathrm{LiTaO}_{3}$ & 20 & 0.015 \\
$3 m,[010]$ & $\mathrm{LiNbO}_{3}$ & 60 & 0.03 \\
$3 m,[001]$ & $\mathrm{LiTaO}_{3}$ & 190 & 0.15 \\
$3 m,[001]$ & $\mathrm{LiNbO}_{3}$ & 80 & 0.10
\end{tabular}

The artificial pyrocoefficients are of the same order of magnitude as the usual pyroelectric coefficients but the voltage sensitivity of piezoelectrics may be amplified many times so in static as in dynamic cases [6]. Consequently, the piezoelectric crystals significantly extend the number of pyroelectric materials and the possibilities of their applications. The "pyroelectric" detector based on piezoelectric crystal in the form of thin disk or long rectangular rod could be simultaneously a microwave dielectric resonator tuned to the main frequency and piezoelectric resonator tuned onto the frequency of modulations $[1,6]$.

The place of two new effects amidst the known effects is shown in Table II. Traditional piezoeffect is possible in 20 piezoelectric (non-central) classes of crystals and is induced by mechanical force. If the excitation is one-dimensional, a longitudinal piezoeffect could be obtained. Volume piezoeffect is the electrical response (vectorial) on the hydrostatic action (scalar). Such a response is possible only in a polar crystal if it is free. But we have already shown that volume piezoelectric response could be obtained in any piezoelectric crystal if it is partially clamped (Table I). Artificial piezoeffect may be induced in all types of solid dielectrics due to electric bias field which produces the polar axis even in scalar medium.

The symmetry of traditional pyroeffect coincides with the volume piezoeffect, therefore an artificial pyroelectricity could be produced by electrical bias. A thermopolarization effect is possible in any solid dielectric, but this effect is very small.

Secondary pyroelectricity produced by strains looks like thermopiezoelectricity but it is inherent only to 10 pyroelectric classes of crystals. It may be observed both in partially clamped condition and in stress-free crystals. Apparently, these 
TABLE II

Classification of electrical responses in crystals.

\begin{tabular}{|c|c|c|}
\hline \multicolumn{3}{|c|}{ Charge separation in insulators } \\
\hline Induced by stress & & Induced by temperature \\
\hline $\begin{array}{l}\text { Piezoelectric } \\
\text { response }\end{array}$ & & $\begin{array}{l}\text { Pyroelectric } \\
\text { response }\end{array}$ \\
\hline $\begin{array}{l}\text { traditional } \\
\text { piezoeffect }\end{array}$ & $\begin{array}{c}\text { New elfects } \\
\text { in } 20 \text { classes of } \\
\text { partially clamped } \\
\text { crystals }\end{array}$ & $\begin{array}{l}\text { traditional } \\
\text { pyroeffect }\end{array}$ \\
\hline longitudinal & termopiezoelectric & secondary \\
\hline piezoeffect & response & pyroeffect \\
\hline 20 classes & 20 classes & 10 classes \\
\hline volume & volume & tertiary \\
\hline piezoeffect & piezoeffect & pyroeffect \\
\hline 10 classes & 20 classes & 20 classes \\
\hline flexoelectric & & thermopolarization \\
\hline effect & & effect \\
\hline 32 classes & & 32 classes \\
\hline artificial & & artificial \\
\hline piezoeffect & & pyroelectricity \\
\hline 32 classes & & 32 classes \\
\hline
\end{tabular}

are the spontaneous strains that play a role of internal "partial clamping" in usual pyroelectrics. It is interesting to note that in partial clamping conditions the secondary pyroelectricity decreases while TPER without these conditions is impossible. Both TPER and secondary pyroelectricity are impossible in totally clamped crystals. It is particularly interesting to compare TPER and tertiary pyroelectricity [7]. Both of them are inherent to piezoelectric classes of crystals and may be excited by elastic stresses which are thermally induced. Nevertheless, the reason of tertiary effect is the spatial inhomogeneity of temperature distribution while the source of TPER is the anisotropy of boundary conditions. The latter allows the possibility of only one type of thermal strains, just in the direction of piezoelectric axis. The other strains are forbidden.

Consequently, under special boundary conditions any piezoelectric is liable to give a vectorial response by the action of scalar influence, therefore the uniform change of temperature causes a sort of "pyroelectricity" named thermopiezoelectric response. 


\section{References}

[1] L.P. Pereverzeva, Yu.M. Poplavko, S.K. Sklarenko, A.G. Chepilko, Pis'ma Zh. Eksp. Teor. Fiz. 3, 820 (1990).

[2] Yu.M. Poplavko, L.P. Pereverzeva, V.F. Żavorotni, S.K. Sklarenko, A.G. Chepilko, Ferroelectrics 131, 331 (1992).

[3] Yu.M. Poplavko, L.P. Pereverzeva, J. Tech. Physs. (Russ.) 62, 93 (1992).

[4] Yu.M. Poplavko, L.P. Pereverzeva, Ferroelectrics 130, 361 (1992).

[5] L.P. Pereverzeva, Yu.M. Poplavko, S.K. Sklarenko, A.G. Chepilko, V.F. Zavorotnyi, Fiz. Tverd. Tela 34, 281 (1992).

[6] L.P. Pereverzeva, Yu.N. Bondarenko, Yu.M. Poplavko, Radioelectronica Izvestia Vuzov (Russ.) 33, 19 (1990).

[7] V.F. Kosorotov, L.S. Kremenchugskii, L.V. Levash, L.V. Shchedrina, Ferroelectrics 70, 27 (1986). 